\title{
The applicability range of the Modified Source Multiplication (MSM) method tested in the fast VENUS-F reactor
}

\author{
Krása $A^{1}$, Kochetkov $A^{1}$, Messaoudi $N^{1}$, Vittiglio $\mathbf{G}^{\mathbf{1}}$, and Wagemans $\mathbf{J}^{1}$ \\ ${ }^{1}$ SCK $\cdot$ CEN, Belgian Nuclear Research Centre \\ Boeretang 200, 2400 Mol, Belgium
}

\begin{abstract}
akrasa@sckcen.be, akochetk@sckcen.be,nmessaou@sckcen.be,gvittigl@sckcen.be, jwageman@sckcen.be
\end{abstract}

\begin{abstract}
The MSM method is an experimental technique for determination of reactivity of a subcritical reactor. It consists of one dynamic measurement followed by two static measurements, which use an extraneous neutron source. For the data analysis, the core averaged kinetic parameters need to be calculated as well as a spatially-dependent correction factor that corrects for the point kinetics approximation. In order to test the range for which the method is valid and to demonstrate the reliability of the correction factor calculations in a fast reactor, a dedicated experimental campaign was performed in the fast lead-bismuth VENUS-F reactor. The reactivity of a dozen of sub-critical configurations was measured with the MSM method using ten ${ }^{235} \mathrm{U}$ fission chambers. The detectors were located at various distances from the active zone and from the extraneous neutron source, leading to a large range of values of a correction factor (calculated with the Monte Carlo MCNP5 code) used in the data analysis.
\end{abstract}

KEYWORDS: MSM method, reactivity, VENUS-F, sub-critical fast core, Monte Carlo method

\section{INTRODUCTION}

The Modified Source Multiplication (MSM) method* [1] is an experimental technique for determination of reactivity of a sub-critical reactor or a reactivity effect. It needs to start from a critical core into which a small negative reactivity is inserted. The reactivity of this reference sub-critical configuration is determined using point kinetics. Then, an extraneous neutron source is inserted into the reference subcritical configuration and the detector count rate is measured. Finally, changes are made to the reactor resulting in a new configuration with unknown reactivity that is to be determined. The extraneous neutron source is inserted again and new detector count rate is measured. The ratio of the two reactivity values is proportional to the ratio of the inverse detector count rates measured in these two reactor configurations.

The MSM method is based on a point kinetics model assuming the neutron flux distribution equals the fundamental mode of the diffusion equation and its shape stays the same in different sub-critical states. In reality, local reactivity insertion disturbs the neutron importance field, fundamental mode distribution and

\footnotetext{
* Sometimes also called Neutron Source Multiplication method (NSM) [2], Neutron Source Multiplication Method (NSMM) [3] or the Modified Neutron Source Method (MNSM) [4].
} 
the higher modes of the neutron source have non-negligible impact. Various approaches to the determination of the corrections were developed, either extracting the fundamental mode and calculating spatial corrections for perturbations [2] or expanding the neutron flux distribution in a sub-critical state in a series of higher mode fluxes of the critical state [3]. In all these cases, calculations were performed using deterministic neutron transport codes.

For the first time, the Monte Carlo MSM method was recently applied for determination of the reactivity of the VENUS-F sub-critical reactor core [5] and its control rod worth [6]. VENUS-F is a fast, leadbismuth, zero-power reactor $[7,8]$ located at the Belgian Nuclear Research Center SCK $\cdot$ CEN. The MSM method is there being applied as a reference method (either with a radio-isotope neutron source [5] or accelerator-based $14 \mathrm{MeV}$ dT source [9]) for the validation of the reactivity monitoring methodology [1012], which will be used in the MYRRHA ADS (Accelerator Driven System) [13].

The Monte Carlo MSM method utilizing a modified MCNP code [5] for calculating the MSM correction factors $f_{\text {MSM }}$ was thoroughly validated in the first VENUS-F sub-critical configuration (rather symmetric and without any perturbations) with a high precision 3D geometry model as well as a simplified one [5]. Standard experimental recommendations were fulfilled [1], namely the reactivity of the reference subcritical configuration was chosen to be well below (in absolute terms) $1 \$$ and the detectors were not located too close to perturbations and the neutron source. It was observed that the $f_{\text {MSM }}$ factor is the biggest near the neutron source, while there are regions far from the neutron source where the correction factor vanishes (equals 1). However, in some of the following VENUS-F configurations it was found that strong perturbations can cause the simplified geometry model to fail and the $f_{\mathrm{MSM}}$ factor does not equal 1 anywhere in the core.

In the present paper, we present results of a new experimental campaign performed at the VENUS-F reactor in 2019 dedicated to test:

- the range of the reactivity of the reference sub-critical configuration for which the method is valid (varied between -0.1 and $-1.4 \$$ );

- the sensitivity of the reactivity measurement to the location of the insertion of the initial small negative reactivity (rod drops in six different locations);

- the reliability of the correction factor calculations even in situations when the detectors are located in positions close to the source or close to the perturbation (ten fission chambers located in the active zone/reflector interface, in the inner reflector and outer reflector);

- the sensitivity of the method to the accuracy of the extraneous neutron source axial position.

We used the MSM method to determine the reactivity of two safety rods that were inserted close to and far away from the rod drop location as well as at different distances from the extraneous Am-Be neutron source. The space-dependent $f_{\text {MSM }}$ factors were calculated using the Monte Carlo MCNP5 code [14].

In section 2, the core configurations assembled for the MSM measurements reported in this paper and the utilized instrumentation are presented. In section 3, the way the MSM method is applied at the VENUS-F reactor is described, the details of the correction factor calculations are given and the uncertainty analysis is elaborated. The experimental reactivity of the reference sub-critical configuration and the reactivity of the safety rods are presented and compared with calculations in section 4 .

\section{THE EXPERIMENTAL SETUP}

The VENUS-F vessel $(160 \mathrm{~cm}$ in diameter) contains a square lattice $(100 \mathrm{~cm}$ side length) of $12 \times 12$ assemblies surrounded with lead reflector, see Fig. 1. To maximize the spatial effects influencing the MSM method, an asymmetric configuration (CC12 core) was loaded. The active zone contained 34 fuel assemblies (30 wt.\% uranium fuel, solid $\mathrm{Pb}+\mathrm{Bi}$ coolant simulator [8]) and six safety rods (SR) that consist 
of a $\mathrm{B}_{4} \mathrm{C}$ absorber part and fuel follower. In the critical $\mathrm{CC} 12$ configuration, the fuel follower of all SRs was at the height of the active zone (SRs OUT). During the MSM measurements, SR1 and SR6 were alternately inserted in the core (SR1 IN or SR6 IN), i.e. the $\mathrm{B}_{4} \mathrm{C}$ part was at the height of the active zone, see right part of Fig. 1. Some fuel assemblies were directly surrounded by lead reflector assemblies, some by graphite reflector assemblies.

A rod drop ( $\mathrm{RD}$ ) system made of $\mathrm{B}_{4} \mathrm{C}$ powder was loaded in six different positions, either instead of a graphite or a lead assembly. The rod drop positions were chosen such to cover a broad range of reactivity values (closer or further with respect to the active zone) and to be located close to SR1 or SR6. Subcritical measurements were performed using an Am-Be source inserted in the lead reflector outside the square lattice of the assemblies, see left part of Fig. 1.

The measurements were performed using ten ${ }^{235} \mathrm{U}$ fission chambers $(0.01-1 \mathrm{~g}$ deposit mass, $12-21 \mathrm{~cm}$ deposit length, 2.5-4.7 cm deposit diameter [15]) placed in experimental channels in graphite reflector assemblies at the active zone/reflector interface as well as at further distances and in lead reflector assemblies, see left part of Fig. 1. Vertically, the middle of the detector deposits coincided with the core mid-plane.

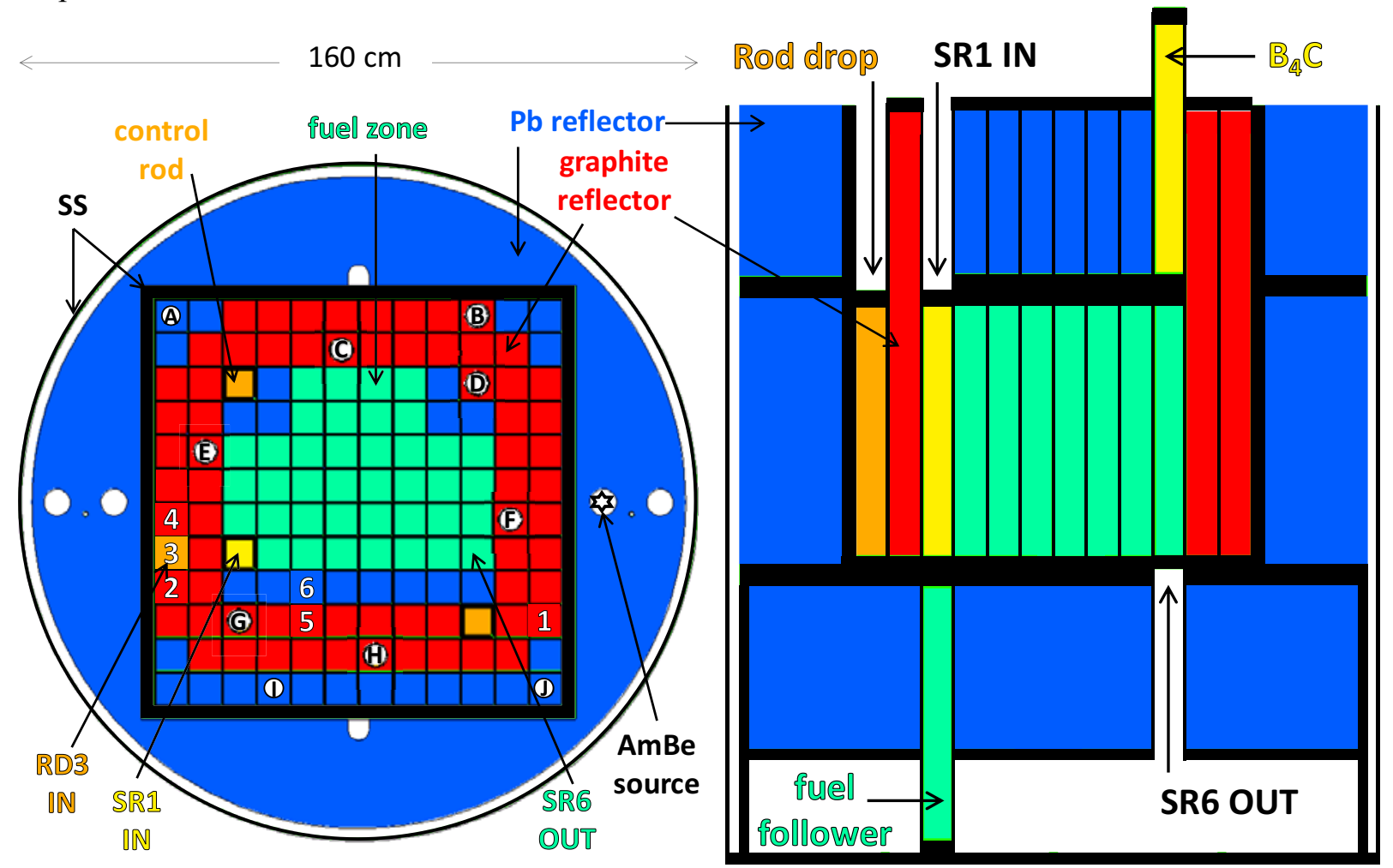

Figure 1. Left: horizontal view through the core mid-plane of the VENUS-F CC12 core with SR1 and rod drop (in location No. 3) inserted. The six optional locations of the rod drop system are indicated with white numbers (1-6). The ten detectors are indicated with black letters (A-J). Right: vertical view of the VENUS-F CC12 core through the plane passing the extracted safety rod SR6, inserted SR1 and rod drop. 


\section{THE MSM METHOD}

\subsection{Experimental Procedure}

The MSM method consists of one dynamic measurement followed by two static measurements. It requires the following procedure.

1. Operate a reactor in a critical state $\left(\rho_{0}=0\right)$ at a stable power for sufficiently long time for neutron population and precursor concentrations reach equilibrium. Measure the static detector count rate $n_{0}$.

2. Insert a small negative reactivity (in our case a rod drop). Measure the detector count rate $n(t)$ as a function of time after the rod drop until counting only background counts (i.e. complete decay of precursors).

3. Determine the reactivity of the reference sub-critical configuration $\rho_{1}$ using the integral counting method (the inverse point kinetics formula leads to the same result [5]):

$$
-\frac{\rho_{1}}{\beta_{\text {eff }}}=\frac{n_{0} \sum_{i=1}^{G} \frac{\alpha_{i}}{\lambda_{i}}}{\int_{0}^{\infty} n(t) d t} .
$$

The spectrum-averaged effective delayed neutron fraction $\beta_{\text {eff }}(738 \pm 2 \mathrm{pcm}$ for the CC12 VENUS-F core) and the relative neutron fractions of each delayed neutron group $\alpha_{i}=\beta_{i} / \beta_{\text {eff }}$ characterized by the decay constants $\lambda_{i}$ were calculated with MCNP5 and JEFF-3.1.2 library [16] with $G=8$ delayed neutron groups (details described in [17]).

4. Insert an extraneous neutron source into the reactor (in our case an Am-Be source) and measure static detector count rates $C_{1}$ in a reference configuration with reactivity $\rho_{1}$.

5. Make the desired change in the core (in our case insert a safety rod), insert the extraneous neutron source again and measure static detector count rates $C_{2}$ in a new sub-critical configuration with unknown reactivity $\rho_{2}$ that is to be determined.

6. Calculate the correction factors $f_{\mathrm{MSM}}$ (for each detector) that connect the two sub-critical configurations in order to account for source importance and detector efficiency changes.

7. Determine the unknown reactivity of the new sub-critical configuration:

$$
\rho_{2}=\rho_{1} f_{\mathrm{MSM}} \frac{C_{1}}{C_{2}}
$$

8. Determine the value of the investigated reactivity effect (in our case a safety rod worth):

$$
\text { SR worth }=\rho_{2}-\rho_{1} \text {. }
$$

\subsection{Correction Factor Calculation}

Before the calculation of the $f_{\mathrm{MSM}}$ correction factors the Monte Carlo neutron transport code was adjusted to get $k_{\text {eff }}=1$ (with a precision of a few pcm) for the initial critical configuration. The MCNP calculation of VENUS-F critical cores overestimates the $k_{\text {eff }}$ value by several hundreds of $\mathrm{pcm}$ [18]. This was solved by modifying the $\bar{v}$ in the MCNP calculation [5] (dividing it by the calculated $k_{\text {eff, }}$ which was $1.00344 \pm$ 0.00002 in case of the critical CC12 core). The correction factor was then calculated as

$$
f_{\mathrm{MSM}}=\frac{\rho_{2, \mathrm{c}}}{\rho_{1, \mathrm{c}}} \frac{C_{2, \mathrm{c}}}{C_{1, \mathrm{c}}},
$$

where each of the four terms is a result of a separate calculation (index "c" indicates the quantity was calculated). The reactivity $\rho_{1, \mathrm{c}}$ and $\rho_{2, \mathrm{c}}$ were results of two KCODE criticality calculations, while the count rates $C_{1, \mathrm{c}}$ and $C_{2, \mathrm{c}}$ were results of two source driven calculations. 
The $C_{1, \mathrm{c}}$ and $C_{2, \mathrm{c}}$ count rates were calculated as the ${ }^{235} \mathrm{U}$ fission rates (F4 tally $+\mathrm{FM}$ multiplier card) in detector cells of cylindrical shape having the outer dimensions of the deposits (see section 2). It was also verified that the detector tally (F5) in MCNP gives identical results (within the statistical uncertainties).

\subsection{Uncertainty analysis}

All quantities in eq. (2) are considered uncorrelated and the uncertainty of the reactivity effect was determined as a square root of the sum of squares of the relative uncertainties of the individual terms. The rod drop measurement was performed several times at different power levels in order to avoid detector dead-time and provide sufficient count rates resulting in uncertainties of counting statistics better than $0.3 \%$ for $n_{0}$ and $n(t)$ in eq. (1). The $1.4 \%$ uncertainty on the delayed neutron parameters [17] is the leading term of the $\rho_{1}$ uncertainty. Both static measurements were performed for sufficiently long time so the uncertainties of counting statistics (i.e., $C_{1}$ and $C_{2}$ ) are less than $0.5 \%$ for all detectors (except those placed far from the active zone, for which $1-2 \%$ was reached).

The uncertainty of $f_{\text {MSM }}$ was calculated by adding the uncertainties of the four terms in eq. (4) in quadrature. Among the four calculations, the source-driven calculation of the reference sub-critical configuration was the most time consuming due to long fission chains near criticality. Therefore, the uncertainty in $C_{1, \mathrm{c}}$ was the major contributor (up to $2.2 \%$ ). The uncertainty in $\rho_{1, \mathrm{c}}$ was also important because the minimal uncertainty in $k_{\text {eff }}$ reasonably achievable with Monte Carlo calculation is 2 pcm, which brought $2.4 \%$ uncertainty in case of the least sub-critical reference sub-critical configuration. The uncertainties in $\rho_{2, \mathrm{c}}$ and $C_{2, \mathrm{c}}$ were negligible $(<0.4 \%)$. The total uncertainty of the measured safety rod worth was between $2 \%$ and $4 \%$.

\section{THE RESULTS}

\subsection{Reactivity of the reference sub-critical configuration}

The rod drop reactivity measured in six locations covered a range of $\rho_{1}$ between -90 and $-1040 \mathrm{pcm}$ (or -0.12 to $-1.4 \$$ ). Each rod drop caused a neutron flux perturbation in the surrounding assemblies. The bigger the rod drop reactivity, the larger the perturbation in the core and the smaller the number of detectors that could be used in the data analysis. In all cases, detectors located at a distance of three assemblies or less from the rod drop were excluded. For heavier rod drops, all detectors located on the same side from the active zone as the rod drop had to be excluded from the data analysis, see Table I.

On the other hand, the magnitude of the perturbation in the detectors close to rod drop was similar for any rod drop reactivity, see left part of Fig. 2. Even in case of the rod drop with $-1.4 \$$, most of the detectors gave consistent results. In general, if fuel assembly was located between rod drop and detector, such detector was effectively shielded from the perturbation caused by the inserted rod drop and could be used in data analysis.

Table I. The experimental rod drop reactivity averaged over a selected set of detectors (for the rod drop location, see left part of Fig. 1).

\begin{tabular}{|c|c|c|c|c|c|c|}
\hline & RD1 & RD2 & RD3 & RD4 & RD5 & RD6 \\
\hline$\rho_{1}[\mathrm{pcm}]$ & $-89.0 \pm 1.5$ & $-180 \pm 3$ & $-276 \pm$ & $-347 \pm$ & $-599 \pm 11$ & $-1033 \pm 23$ \\
\hline$\rho_{1}[\$]$ & $-0.121 \pm 0.002$ & $-0.244 \pm 0.004$ & $-0.374 \pm 0.007$ & $-0.470 \pm 0.008$ & $-0.812 \pm 0.015$ & $-1.40 \pm 0.03$ \\
\hline \# detectors & 8 & 7 & 7 & 6 & 6 & 6 \\
\hline StDev & $0.8 \%$ & $0.7 \%$ & $1.2 \%$ & $0.7 \%$ & $1.0 \%$ & $1.7 \%$ \\
\hline
\end{tabular}



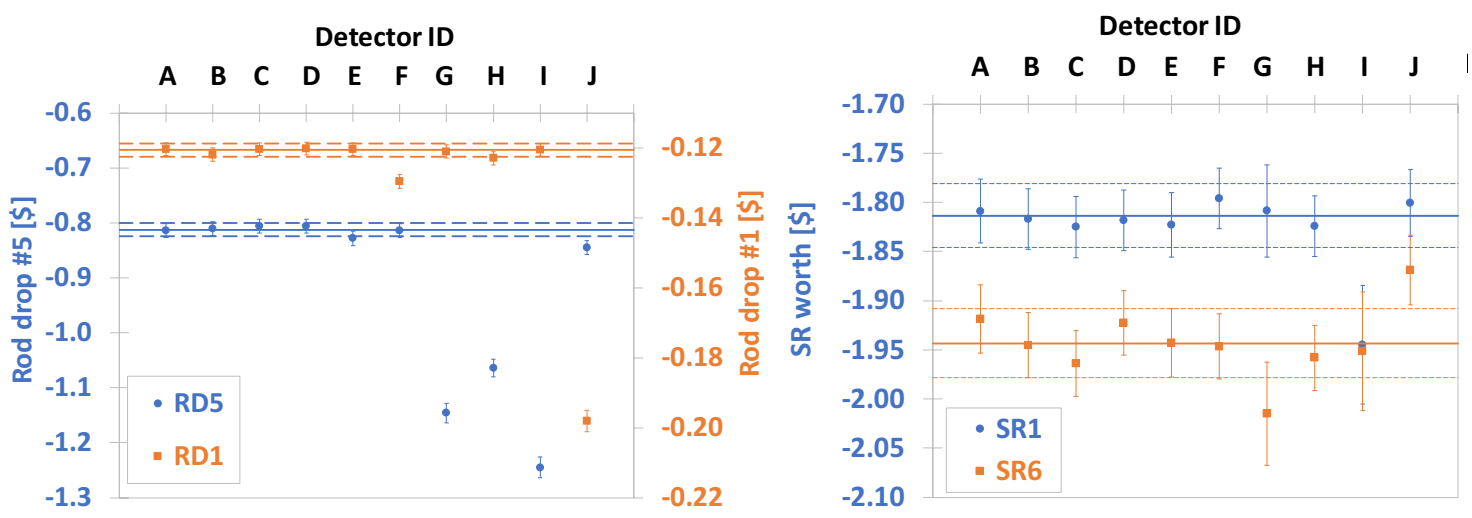

Figure 2. Left: Rod drop in positions 1 and 5 measured with ten detectors (for the detector locations, see left part of Fig. 1). The solid and dashed lines represent average and $1 \sigma$ uncertainties (excluded detectors: F, J for RD1, G, H, I , J for RD5).

Right: SR1 and SR6 worth measured with ten detectors and using RD5 as a reference sub-critical configuration. The solid and dashed lines represent average and $1 \sigma$ uncertainties.

\subsection{Safety rod worth}

The worth of the safety rods in VENUS-F is rather big (around $-2 \$$ ) due to a combined effect of absorber insertion and fuel follower extraction. SR1 and SR6 were chosen for the MSM measurement because they are located each at opposite side of the active zone, thus, being sensitive to different core components. SR1 was close to RD2 - RD6, while SR6 was close to RD1 and the Am-Be source.

Measurements using the ten detectors gave exclusively consistent results and all detectors could be used in data analysis. This observation gives confidence in the calculated correction factors, which had values between 0.77 and 1.46 for SR1 and between 0.77 and 0.96 for SR6, see Fig. 3. Although the reactivity of the reference sub-critical configuration spans over a large range, the $f_{\text {MSM }}$ factors do not differ much for a single detector used with various reference configurations. There are even detectors with almost identical value (differences less than $2 \%$ ) of $f_{\text {MSM }}$ when compared among the six reference sub-critical configurations (e.g. detectors $\mathrm{A}$ and $\mathrm{H}$ for the SR1 measurement). It indicates low sensitivity of the $f_{\mathrm{MSM}}$ factor calculation to the accuracy of the rod drop reactivity in MCNP calculations.

As expected, the SR worth is influenced by the rod drop. One of the main aims of this work was to investigate whether there is any minimal rod drop reactivity value below which the measured SR worth is free of the rod drop influence. SR worth turned out to be rather sensitive to the rod drop location and much less sensitive to the rod drop reactivity value, see left part of Fig. 4. The absolute value of SR1 worth increases with increasing distance from the rod drop (and with a decreasing absolute value of the rod drop reactivity) from $(-1.68 \pm 0.03) \$$ to $(-2.05 \pm 0.08) \$$ (difference of $18 \%)$. However, the increase is not monotonic as the SR1 worth is the same (within uncertainties) in case of RD5 and RD4 as well as RD3 and RD2.

The SR6 worth varies very little (less than $5 \%$ difference) in case of the rod drop located far from SR6 (i.e. RD2 - RD6). The absolute value of SR6 worth reduces in case of RD1 that was located just $11 \mathrm{~cm}$ from SR6. We therefore conclude that for the MSM measurement of a reactivity effect (as SR worth), the rod drop should be inserted on the other side of the fuel zone than the reactivity effect takes place. Otherwise, the absolute value of the reactivity effect is suppressed (shielded) by absorption in the rod drop material. 

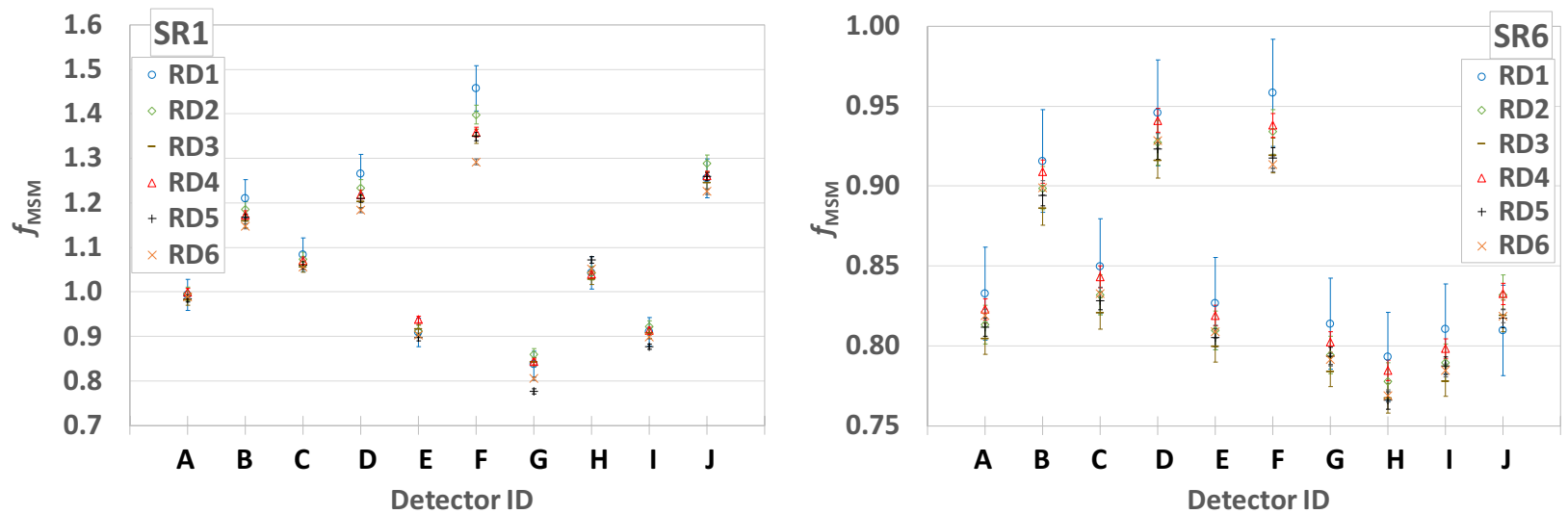

Figure 3. MSM correction factors for SR1 worth (left) and SR6 (right) measured with respect to six different reference sub-critical configurations.

\subsection{Comparison of experiments and calculations}

The measured rod drop, SR1 and SR6 worth are compared with MCNP calculations in Fig. 4. The precision of reactivity effect calculations with absolute values above $500 \mathrm{pcm}$ can be easily made negligible using Monte Carlo method. The worth of SR1 and SR6 was calculated with a precision of $0.2 \%$. The uncertainty of $\mathrm{C} / \mathrm{E}$ (calculation-to-experiment) ratio is thus fully dominated by the experimental uncertainty. In case of a rod drop, the MCNP uncertainty was up to $3.5 \%$ and the $\mathrm{C} / \mathrm{E}$ have thus larger error bars, see right part of Fig. 4.

MCNP slightly underestimates the rod drop and SR worth (by 4-10\%) and it correctly describes the experimental trends. The only exception is the SR1 worth measured with respect to RD3, where MCNP underestimates the experiment by $14 \%$ and predicts bigger value than for SR 1 measured with respect to $\mathrm{RD} 4$, contrary to the experiment. The reason is unclear and it is planned to repeat this measurement.
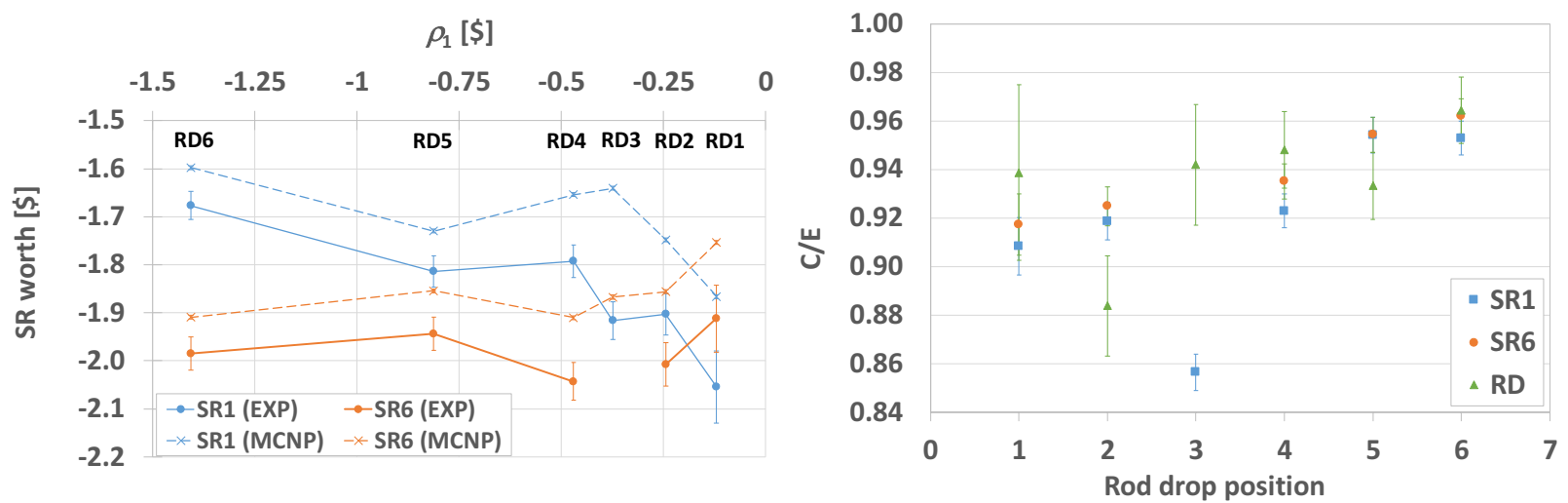

Figure 4. Left: Experimental and calculated SR worth as a function of the reference sub-critical configuration reactivity (SR6 + RD3 was not measured yet, this point will be added in an update).

Right: Ratio of MCNP calculated values and measured values for SR1, SR6 and rod drop.

\section{CONCLUSIONS}

We did not confirm the earlier requirement for the absolute value of the reference sub-critical configuration reactivity to be less than 1 \$. Indeed, heavier rod drop means larger perturbation and less 
detectors can be used in data analysis. However, for the rod drop reactivity $\left|\rho_{1}\right|<1.4 \$$, all detectors give consistent results if they are placed on the opposite side of the fuel zone than the rod drop. Advantage of heavier rod drop was observed as it enables to reduce the Monte Carlo calculation time of the MSM correction factors. To control the validity of the MSM method, the detectors were placed in such a way that a large range of MSM correction factors could be used in the data analysis. We observed excellent agreement of corrected results from detectors located anywhere in the core, even close to perturbations and neutron source. Even a very light $\operatorname{rod}$ drop $\left(\rho_{1} \approx-0.1 \$\right)$ changes the value of a reactivity effect inserted at the same side of the active zone as the rod drop. Therefore, the rod drop should be inserted on another side of the fuel zone than the reactivity effect of interest takes place.

\section{REFERENCES}

1. P. Blaise, F. Mellier, P. Fougeras, "Application of the Modified Source Multiplication (MSM) Technique to Subcritical Reactivity Worth Measurements in Thermal and Fast Reactor Systems," IEEE Transactions On Nuclear Science, 58 (3), pp. 1166-1176 (2011).

2. M. Tsuji, N. Suzuki, Y. Shimazu, "Subcriticality Measurement by Neutron Source Multiplication Method with a Fundamental Mode Extraction," J. Nucl. Sci. Techn. 40 (3), pp. 158-169 (2003)

3. T. Misawa, H. Unesaki, "Measurement of Subcriticality by Higher Mode Source Multiplication Method," Proceedings of ICNC2003, Tokai (Japan), 20-24 Oct 2003, JAERI-Conf 2003-019, pp. 178$182(2003)$.

4. G. Truchet et al., "Application of the modified neutron source multiplication method to the prototype FBR Monju," Annals of Nuclear Energy, 51, pp. 94-106 (2013).

5. J. L. Lecouey et al., "Estimate of the reactivity of the VENUS-F subcritical configuration using a Monte Carlo MSM method," Ann. Nucl. Energy 83, pp. 65-75 (2015).

6. J. L. Lecouey et al., "Monte Carlo MSM correction factors for control rod worth estimates in subcritical and near-critical fast neutron reactors," EPJ Nuclear Sci. Technol. 1 (2), pp. 1-9 (2015).

7. A. Kochetkov et al., "The Lead-Based VENUS-F Facility: Status of the FREYA Project," EPJ Web of Conferences 106, 06004 (2016).

8. A. Krása et al., "The VENUS-F Reactor for Nuclear Data Validation," Proceedings of ISRD-16, Santa Fe (USA), 7-12 May 2017, ASTM STP1608, pp. 286-295 (2018).

9. A. Billebaud et al., "Extended MSM method to estimate the reactivity of a sub-critical core driven by an accelerator based neutron source," Proceedings of PHYSOR-2020, Cambridge (UK), Mar 29-Apr 32020.

10. J. L. Lecouey et al., "Reactivity Measurement with Threshold Fission Chambers in the MYRRHA Mock-up Fast Subcritical Reactor VENUS-F," Proceedings of PHYSOR-2018, 102853 (2018).

11. N. Marie et al., "Reactivity monitoring of the accelerator driven VENUS-F subcritical reactor with the "current-to-flux" method," Annals of Nuclear Energy 128, pp. 12-23 (2019).

12. A. Kochetkov et al., "Source Jerk Integral Method For Sub-Criticality Measurements In ADS," Proceedings of PHYSOR-2020, Cambridge (UK), Mar 29-Apr 32020.

13. G. Van den Eynde et al., "An updated core design for the multi-purpose irradiation facility MYRRHA," Journal of Nuclear Science and Technology 52 (7-8), pp. 1053-1057 (2015)

14. X-5 Monte Carlo Team, "MCNP - A General Monte Carlo N-Particle Code, Version 5," LANL LAUR-03-1987 (2003)

15. J. Wagemans et al., "Nuclear instrumentation in VENUS-F," EPJ Web of Conferences 170, 04027 (2018).

16. A. Santamarina, D. Bernard, Y. Rugama, “The JEFF-3.1.1 Nuclear Data Library," JEFF Report 22 (2009).

17. A. Krása et al., "Impact of delayed neutron constants on reactivity effects measured in a fast reactor," EPJ Web of Conferences 211, 03004 (2019).

18. A. Krása et al., "Comparative study on neutron data in integral experiments of MYRRHA mockup critical cores in the VENUS-F reactor ," EPJ Web of Conferences 146, 06019 (2017). 